\title{
Karies gezielt bekämpfen
}

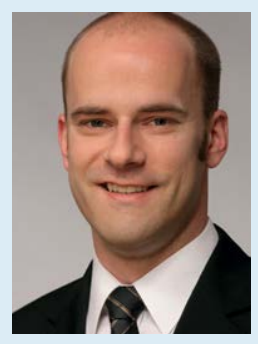

Dr. Joachim Elzer, Project Manager Scientific Affairs bei CP GABA GmbH
Dr. Joachim Elzer hat an der Universität Heidelberg in Molekularbiologie promoviert. Er blieb der Forschung nach Abschluss seiner Doktorarbeit noch drei Jahre treu und wechselte dann in die Praxis. Seit 2013 ist Elzer Project Manager Scientific Affairs bei CP GABA und arbeitet in den Themenfeldern Karies und Kinderzahnheilkunde.

? Herr Dr. Elzer, Sie waren viele Jahre in der Forschung tätig. Welchen Nutzen hat Ihr wissenschaftlicher Hintergrund in Ihrer jetzigen Tätigkeit als Project Manager bei CP GABA?

Dr. Elzer: Die Wirksamkeit aller Mundhygiene-Produkte von CP GABA wird klinisch getestet und bestätigt. Die Erkenntnisse und Vorteile unserer Produkte, die wir aus der klinischen Forschung gewinnen, müssen wir in eine für den Konsumenten freundliche Sprache übersetzen. Da ist es natürlich von großem Vorteil, wenn man bereits selbst wissenschaftlich gearbeitet hat.

? Sie beschäftigen sich im Rahmen Ihrer Tätigkeit bei CP GABA intensiv mit Karies. Ist Karies in unseren Breitengraden nicht längst besiegt?

Dr. Elzer: Tatsächlich konnte die Kariesprävalenz in den letzten Jahren reduziert werden. Mit den Fluoriden, die in Zahnpasten, Mundspülungen und Gelen enthalten sind, haben wir einen sehr guten chemischen Ansatz gegen Karies. Aber Karies ist noch lange nicht besiegt. 99 Prozent aller Erwachsenen haben eine Karieserfahrung. Zudem besteht das Kariesrisiko das ganze Leben lang. Nur regelmäßige, andauernde Pflege - am besten mit fluoridhaltigen Zahnpflegeprodukten - kann vorbeugen.

? Welche Personengruppen sind besonders kariesgefährdet und warum?

Dr. Elzer: Besonders gefährdet sind Kinder und ältere Menschen, allerdings aus ganz unterschiedlichen Gründen. Bei Kindern ist der Zahnschmelz der Milchzähne schlicht anfälliger, bei älteren Menschen erhöhen der geringere Speichelfluss und gegebenenfalls eingeschränkte motorische Fähig- keiten das Kariesrisiko. Außerdem sind Personen, die häufig Zwischenmahlzeiten zu sich nehmen oder eine schlechte Mundhygiene haben, einem erhöhten Risiko ausgesetzt, da diese Verhaltensweise das Wachstum schädlicher Bakterien fördert.

? Wie kann man Karies weiter zurückdrängen und insbesondere die Kinder zu einer guten Mundhygiene erziehen?

Dr. Elzer: Damit die Kariesprävalenz in den nächsten Jahren weiter abnimmt, brauchen wir Mundhygienekonzepte, die speziell auf die Risikogruppen zugeschnitten sind. Das alleine reicht aber nicht. Die Anleitung für eine gute Zahnpflege muss auch über eine zielgerichtete Kommunikation bei den Risikogruppen ankommen. Im Falle der Frühkindlichen Karies sollten die Kinder etwa spielerisch und mit Fantasie an eine gründliche und regelmäßige Zahnpflege herangeführt werden. Die Ansprache und Informationsvermittlung muss indirekt erfolgen: über die Kinderärzte, Erzieher, Hebammen und natürlich auch die Eltern und Erziehungsberechtigten - denn sie sorgen letzten Endes für die Zahnpflege der Kinder. CP GABA und die Bundeszahnärztekammer haben gerade im Rahmen der „Initiative für eine mundgesunde Zukunft in Deutschland“ praxiserprobte Konzepte ausgezeichnet, die sich der Frühkindlichen Karies widmen. Die Arbeit der Initiative wird in den kommenden Jahren fortgesetzt und wird sich dann auch anderen Risikogruppen zuwenden.

? Welche Rolle spielen digitale bzw. soziale Medien im Kampf gegen Karies? Dr. Elzer: Die neuen Medien spielen in unserem beruflichen und privaten Alltag eine immer größere Rolle. Diese Entwicklung macht vor den wichtigsten Ansprechpartnern im Kampf gegen Karies nicht halt. Deswegen unterstützt CP GABA die fachliche Weiterbildung der zahnärztlichen Praxisteams etwa über Webinare und den Prophylaxe Team Club auf Facebook. Zudem fördern wir in der bereits angesprochenen „Initiative für eine mundgesunde Zukunft in Deutschland“ multimediale Konzepte für die Ansprache von Kariesrisikogruppen.
? Auf der diesjährigen ORCA-Tagung in Brüssel wurde Karies von Experten als nicht übertragbare Krankheit bewertet. Was genau bedeutet diese Klassifizierung und welche Folgen hat sie für Prävention und die Behandlung von Karies? Dr. Elzer: Diese neue Klassifizierung hat weitreichende Folgen für die Prävention und Behandlung von Karies. Denn sieht man Karies als übertragbare Krankheit, werden einzelne Bakterienstämme als Erkrankungsursache ausgemacht und in einer Behandlung gezielt bekämpft. Die Klassifizierung von Karies als nicht übertragbare bzw. als chronische Krankheit erkennt multifaktorielle Ursachen an. Im Behandlungsfokus stehen nicht mehr einzelne Bakterienstämme, sondern der gesamte Stoffwechsel im oralen Biofilm. Insofern bestätigen die Experten der ORCA-Tagung den Ansatz von CP GABA. Der Zuckersäuren-Neutralisator ${ }^{\mathrm{TM}}$ basiert auf der PROARGIN $^{\circledR}$-Technologie von CP GABA, in der eine unlösliche Kalzium-Karbonat-Quelle mit dem Wirkstoff Arginin kombiniert wird. Durch den bakteriellen Abbau von Kohlenhydraten im Mundraum entstehen Säuren, die wiederum zu einer Demineralisierung des Zahnschmelzes führen. Der Wirkstoff Arginin, der unter anderem in der Zahnpasta elmex ${ }^{\circledR}$ KARIESSCHUTZ PROFESSIONAL ${ }^{\mathrm{TM}}$ enthalten ist, wird ebenfalls durch Bakterien des Biofilms abgebaut. Allerdings entstehen Basen, die die Säuren neutralisieren und den oralen Biofilm in die Balance bringen. Die Remineralisierung des Zahnschmelzes kann schneller einsetzen.

? Sie sind selbst Vater. Welche Strategie haben Sie verfolgt, um Ihre Kinder zu einer guten Mundhygiene zu bewegen und mit welchem Erfolg?

Dr. Elzer: Mit dem erhobenen Zeigefinger kommt man bei Kindern meist nicht weit. Deswegen haben wir mit dem ersten Zahn versucht, das Interesse und den Spieltrieb der Kinder zu wecken. Wir haben die Kinder selbst putzen lassen, um sie in die Mundhygiene zu integrieren. Wichtig ist aber: Die Eltern sollten dann nochmal gründlich nachputzen.

Diese Rubrik ist entstanden mit freundlicher Unterstützung der CP GABA GmbH, Hamburg 